\title{
Pic de la Mirandole et son mythe, tels que vus par William G. Craven*
}

\section{LOUIS VALCKE}

Le malheureux Pic de la Mirandole n'était plus guère qu'un nom, que l'ironie voltairienne avait un instant tiré de l'obscurité. Jacob Burckhardt, cependant, créa la Renaissance, et Pic, que ses contemporains avaient prophétiquement appelé "il fenice degli ingegni" allait lui aussi renaître. Mais, nous dit William G. Craven, sous condition. Sous condition de se conformer à ce que l'histoire attendait de lui: "that he must have had a philosophy of man as a free individual; that he must have been undermining revealed religion; more generally, that he must have been saying radically new things" (p. 159). Or, ajoute l'auteur, "taken together, these can be recognised as characteristics of what is conventionally known as the Burckhardtian Renaissance" (ibid.). C'est donc dire que lorsque Pic renaîtra, à la fin du siècle dernier, il renaîtra sous la forme d'un mythe, qui sera peut-être très différent du personnage réel qui vécut à Florence à la fin du Quattrocento.

C'est à la mise à nu de ce mythe que se livre W.G. Craven par une critique serrée de chacun des éléments qui constitutent ce qu'il nomme "the accepted picture of Pico" ( $p$. 19). Tous les thèmes qui tissent la toile d'un certain conformisme interprétatif sont passés en revue et confrontés systématiquement à l'examen des textes de Pic. Ce sont "the search for concord and synthesis, the idea of a universal revelation, Pico's rationalism and theological radicalism, and especially the complex of ideas associated with man: his worth, freedom, and mutability and cosmic function" (ibid.).

Craven n'épargne personne, et surtout pas les plus grands, que sont Garin et Cassirer. Reconnaissant évidemment l'importance première et fondamentale de la recherche d'Eugenio Garin, dont il fait explicitement l'éloge (p. 5), il n'en rejette pas moins la plupart des conclusions aux quelles celui-ci avait cru devoir aboutir. Je ne citerai qu'un seul paragraphe, particulièrement représentatif: 
... Garin's reconstruction was a highly imaginative one. ... He was unable to find evidence for the basic presuppositions he suggested, the unity of the intellect or of thought and the coincidentia oppositorum, and was reduced to quoting meaningly from Averroes. His account of the De ente misrepresented its purpose, inflated its importance and misinterpreted its text. The problem of reconciling philosophy and religion must have been deduced from the supposed Averroist influence, because it does not seem to have occurred to Pico. This, in turn, deprives Kabbalah of its place in Garin's scheme, a place which Pico clearly never envisaged for it. The religion of spirit, in whose birth it was to assist, was the product of a thoroughly anachronistic reading of the texts. Finally, his idea of man as the cosmic synthesis was based on a far-fetched reading clouded by presuppositions redolent of Idealism. Many details of Garin's interpretation were inaccurate, and its whole unifying scheme was misconceived. (p. 6)

Ce qui vaut pour Garin vaut, mutatis mutandis, pour tous les autres qui auront souligné ou vont reprendre tel ou tel de ces traits dont l'accrétion va constituer l'image mythique de Pic de la Mirandole: libération de l'homme pour Cassirer, orgueil prométhéen du Mage pour D.P. Walker et Frances Yates, rationalisme religieux et déisme selon Saitta et Pusino, préréformisme pour Kieszkowski, pélagianisme pour Herschel Baker et Enno Van Gelder. Tout cela se produira sous l'influence, prédominante sinon exclusive, du néoplatonisme selon Anagnine; de l'hermétisme selon Yates; de Nicolas de Cues, selon Cassirer; de la Cabale, selon Garin, ou de l'averroïsme selon Bruno Nardi .... .

Le lecteur aura reconnu dans cet échantillonnage quelques-unes des idées les plus répandues et les plus communément admises au sein de la littérature picienne. Tout le livre serait à citer: c'est un à un que sont décortiqués tous les clichés concernant l'oeuvre de Pic et son importance historique. Encore la panorama n'est-il pas complet: manque à l'appel nul moins que Ernst Bloch qui en deux pages de sa Philosophie de la Renaissance amoncèle sereinement toutes les idées reçues qui ont jamais été énoncées à propos de Pic en qui il salue le restaurateur du paganisme grec. ${ }^{1}$ Il y manque également Lidia Braghina, la marxiste de service, qui réussit à voir en Pic le champion de l'accès démocratique à la réflexion philosophique, ${ }^{2}$ ce même Pic auquel Garin adresse le reproche d'être, par son goût de l'ésotérisme et sa passion du secret, quelque peu élitiste: ${ }^{3}$ il est loisible à tout un chacun de ne comprendre l'histoire que sous le seul éclairage de la lutte des classes, encore faut-il prendre soin de ne pas confondre les versants de la barricade... .

Seuls touvent grâce aux yeux de Craven, et encore très relativement, "Dulles, Di Napoli, de Lubac and, to some extent, Monnerjahn ..." (p. 19), pour la raison qu'étant théologiens, ces auteurs ont souligné ce que Pic devait à la scolastique et à la patristique, réduisant d'autant son originalité et allant par le fait même à l'encontre de ce qu'on est en droit d'appeler le conformisme burckhardtien des études rinascimentales. 
C'est donc à une sorte de massacre en règle que l'on assiste tout au long de ce volume. On croira cependant l'auteur lorsqu'il nous affirme que "this study is not intended as an exercise in academic iconoclasm, gleefully catching great scholars making random, and merely human, mistakes" (p. 155). Son intention est autre et d'ailleurs la question n'est pas là: la question est de savoir si l'auteur mène bien son jeu, s'il fonde ses critiques sur une argumentation solide, étayée par une lecture convaincante, et surtout exhaustive des textes de Pic. Or, à cette question la réponse est affirmative, sans réserve. L'enquête est fort bien menée et les résultats parfaitement concluants. D'ailleurs, ce n'est pas diminuer les mérites de cet ouvrage que de dire qu'il devait être écrit, tôt ou tard. Tout lecteur attentif, jetant un regard neuf sur l'ensemble de l'oeuvre de Pic ne peut que faire siennes les conclusions de Craven. Notons que de Lubac, dans son étude sur Pic, lui avait déjà largement ouvert la voie, ${ }^{4}$ et cette remarque ne veut que mieux souligner l'importance du présent ouvrage, qui va beaucoup plus loin, est systématique et presque complet.

$$
* * *
$$

La critique de Craven est tout entière centrée autour de l'interprétation de l'Oratio, ce discours introductif qui forme avec les Conclusiones et l'Apologia une trilogie, dont il fut la partie historiquement la moins importante, tout en en devenant, par après, de loin, la plus célèbre. Tout est parti de ce texte: de toute l'oeuvre de Pic, Burckhardt ne retient que cette citation tant rabachée par après, qui semble reconnaître à l'homme cette liberté et cette plasticité infinies, en lesquelles on voudra voir le symbole de l'homme prométhéen, préfiguration de toutes les libérations futures. L'Oratio cependant est couchée en un style littéraire - ce qui permet toutes les licences de la rhétorique. Malgré cela, on la prit à la lettre, et dès lors, on y vit une déclaration de principe, fruit d'une mûre réflexion et, comme le dit très pertinemment W.G. Craven, on refusa d'admettre "that Pico was speaking metaphorically, of the moral order" (p. 35). Somme toute, on fit de ce discours exhortatif, un traité de métaphysique.

Le problème est que Pic écrivit également cette christologie mystique qu'est l'Heptaplus; l'opuscule métaphysique du De ente et uno, qui joue un rôle crucial dans son oeuvre; les Disputationes, critique rigoureuse de l'astrologie; sans parler de nombreuses oeuvres mineures d'édification religieuse. Ces autres oeuvres, on ne pouvait les ignorer totalement, mais on pouvait les contourner, les détourner, les escamoter, et surtout on pouvait n'en retenir que ce qui cadrait avec l'idée préconçue que l'on se faisait de Pic. Aucun fil conducteur manifeste ne reliant ces oeuvres si diverses, il devenait loisible d'en supposer un - et là, le choix était vaste. C'est ainsi que Pic devint "the mirror-image of the historian's expectations" (p. 161).

D'où résulte évidemment la tendance à donner des interprétations totale- 
ment anachroniques à certains textes, et, même parmi les plus grands, certains n'hésiteront pas, à l'occasion, de traduire de façon biaisée, sinon erronée, les passages gênants .... .5

A quoi vient s'ajouter le poids de l'autorité qui, en quelque sorte, garantissait la validité de telle interprétation privilégiée, que d'autres, sur la foi de cette garantie, allaient reprendre, développer et renforcer: "When a scholar of Cassirer's immense learning and intellectual stature proposes an interpretation, ... it is only natural that others would feel justified in building it into their own broader surveys" (p. 155-156).

Si importante soit-elle, cette mise à nu du mythe de Pic ne constitue pas le but premier de Craven. Parmi les huit chapitres que contient son ouvrage, le premier et le dernier ont en effet une orientation toute différente, puisqu'il ne s'agit plus d'y exposer un mythe, mais bien d'en rendre compte, c'est-à-dire de démonter les mécanismes intellectuels qui l'ont rendu possible. Pour ce faire, Craven adopte la théorie de Quentin Skinner selon laquelle c'est l'attente même de l'historien qui génère une quadruple "mythologie": mythologie quant à la cohérence et à la doctrine, mythologie par "esprit de clocher" ('parochialism') et par prolepse. On entend par ces termes, d'abord, que l'historien des idées présuppose que l'auteur étudié aura fait preuve d'une rigoureuse cohérence interne, cohérence qui l'aura automatiquement conduit à développer une doctrine systématique: on peut, avec Craven, citer le cas d'Avery Dulles qui croit trouver en Pic une ontologie, une cosmologie, une anthropologie, une psychologie et une théologie. La prolepse, d'autre part, consiste à attribuer à un auteur du passé telle intuition ou perspective par lesquelles il aurait anticipé telle conception ou idée qui ne s'épanouiront que beaucoup plus tard. Cette lecture n'est rendue possible que par un certain "esprit de clocher" qui consiste, d'abord, à isoler de son contexte telle remarque de l'auteur, puis, sur la base d'une ressemblance toute superficielle et accidentelle, d'assimiler cette remarque à telle préoccupation propre au milieu et au temps du lecteur. De cette manière, l'historien ne fait que redécouvrir dans un texte ce qu'il y avait lui-même d'abord enfoui. C'est ainsi qu'on lira la thèse condamnée de libertate credendi sous le seul éclairage de notre moderne liberté de conscience - en ne tenant aucun compte du contexte historique de la problématique mirandolienne, et en n'étant dès lors plus capable de comprendre qu'en cette thèse, bien loin d'exalter la puissance de la liberté, Pic en marque au contraire les limites. D'une manière générale, affirme Craven "in their interpretations of the condemned theses, the historians have been all too ready to attribute to Pico the meaning immediately suggested to them by the words as they stand. They have seldom tried to investigate their background, to enquire whether they have a technical mean- 
ing, or to reflect on the likelihood of Pico intending such a meaning in such circumstances" (p. 51-52). ${ }^{6}$

$* \quad * \quad *$

Tels sont les facteurs intellectuels ou psychologiques à travers lesquels s'opéreraient les mythifications. Pour les signifier globalement, Quentin Skinner eut recours à la notion de paradigme qu'il emprunta à Thomas $\mathrm{S}$. Kuhn pour l'appliquer à son propre domaine de recherche, celui de l'histoire de la pensée politique. Prolongeant à son tour la démarche de Skinner, Craven prétend pouvoir donner une nouvelle extension à cette notion, puisqu'il l'applique ici aux interprétations données à l'oeuvre et à la pensée de Pic.

Selon Kuhn, un paradigme est d'abord une nouvelle interprétation d'un ensemble de phénomènes qui, très vite, parce qu'elle réussit, c'est-à-dire parce qu'elle rend plus facilement intelligibles les phénomènes en question, va se substituer aux interprétations antérieures. Transmises au sein de la communauté scientifique, ce mode d'interprétation va s'implanter et élargir son emprise jusqu'à devenir "partly myth and partly dogma, a tradition or a set of received beliefs" (p. 160). Somme toute, le paradigme deviendra une grille de lecture, la seule grille de lecture jugée acceptable par un groupe à une époque donnée. Elle procurera alors "a chart through an otherwise bewildering chaos of data, though of course necessarily at the price of a drastically reduced vision" (p. 160-161).

Etendant ces considérations aux études rinascimentales, Craven défend la thèse selon laquelle la conception générale de Burckhardt y a joué la rôle de paradigme, et c'est ce que notre auteur tente d'illustrer par le cas exemplaire de Pic. C'est en cela que consiste la thèse centrale de l'ouvrage, toutes les analyses préliminaires trouvant ici leur foyer de convergence, car seul le "paradigme burckhardtien" permettrait de comprendre la mythification dont Pic fut victime et l'incroyable faiblesse du sens critique qui a laissé se constituer un tel mythe: "Ideas like paradigm or mental set seem to be the only plausible way of explaining the kind of misrepresentation which I have been criticising, and in particular the neglect of context so frequently encountered" (p. 161).

Craven tire alors la seule conclusion possible: il faut abandonner ces schémas ambitieux qui ordonnent et coordonnent, il faut rechercher dans les oeuvres de Pic ce que Pic lui-même y a mis et pour cela il faudra utiliser ses propres catégories, non les nôtres. Cela va de soi.

Cela va de soi, sauf que Craven éprouve le besoin d'apporter une justification théorique à un tel programme, et après Skinner et Collingwood à qui il en appelle, il croit trouver cette justification dans l'affirmation selon laquelle "il n'y a pas de problèmes pérennes en philosophie, mais seulement des réponses individuelles à des questions individuelles" (p. 163). 
C'est ici que la situation devient plus critique. On peut d'abord se demander si la transposition de la notion de paradigme, hors de son champ d'application initial, est légitime. On le sait, Kuhn lui-même ne prétendait appliquer ce concept qu'à l'histoire des sciences exactes, et le paradigme kuhnien d'origine ne concerne donc que les données et les hypothèses de ces sciences. Or, l'objet des sciences naturelles nous est "étranger," nous est "opaque": à moins d'en revenir à une physique des essences, nous ne prétendons plus pénétrer l'intérieur des choses pour en déceler les natures et pour en mettre à nu la "raison d'être" ou l'intelligibilité propre par le moyen de l'abstraction. A défaut de quoi nous ne pourrons que rendre intelligibles les interrelations entre les choses. Et pour cela nous aurons recours à quelque construction conceptuelle, ce que Kuhn nomme paradigme. Le paradigme est donc, dans les sciences de la nature, un instrument indispendable. Mais sa valeur n'est que relative: nous ne prétendons pas, par son intermédiaire, avoir accès à la vraie nature des choses. Somme toute, nous retrouvons ici le prudent "tout ce passe comme si ..." du positivisme. Du plus, le risque inhérent au paradigme, c'est précisément de méconnaître cette limitation, c'est précisement d'absolutiser le paradigme en lui attribuant une portée ontologique.

La situation est bien différente lorsque la recherche porte sur une oeuvre humaine. Ici, l'opacité de l'objet fait place à une affinité car l'oeuvre ne nous intéresse que comme intermédiaire par lequel peut s'établir une communication entre l'auteur et son lecteur. Quant à prétendre que ce "solipsisme des questions et des réponses" rendrait illusoire la communication, ce n'est là que rhétorique, car prises à la lettre - de virtute sermonis - de telles affirmations ou négations péremptoires rendraient vaine à priori toute recherche historique. Les oeuvres humaines ont, de fait, une signification transhistorique, par l'intersubjectivité qui les relie, et pour justifier cette intersubjectivité point n'est besoin de faire appel à quelque sympathie mystique, englobant l'humanité ni même à quelque identité ou communauté de nature: il suffit de constater une similitude psychologique - que l'on n'expliquera pas ou que l'on expliquera comme on le voudra.

Par conséquent, les concepts explicatifs, les catégories, les "paradigmes" n'auront pas ici, c'est-à-dire dans leur application aux oeuvres humaines, pour fonction de rendre ces oeuvres intelligibles, mais bien d'en révéler l'intelligibilité propre ou inhérente, qui est celle que leurs auteurs y ont mise. Il s'ensuit, d'une part, que le choix de ces concepts explicatifs sera infiniment délicat, puisqu'ils devront être respectueux de l'oeuvre, et cette oeuvre étant toujours aussi marquée par l'individualité de l'auteur, ils devront toujours rester indéfiniment malléables. D'autre part, et à ce prix, ces paradigmes acquerront une signification réelle, non pas ontologique, sans doute, mais anthropologique: il n'y a pas de barrière absolue entre le discours d'un auteur et la compréhension que nous pouvons en avoir. C'est d'ailleurs ce que Craven reconnaît implicitement, sinon on ne comprendrait pas qu'il puisse 
reprendre à son compte cette exhortation de P. Burke: "the cultural historian needs to be able to use the categories of the men he is studying to look for their logic instead of imposing his own" (p. 162).

On conclura que s'ils sont appliqués avec souplesse, $s$ 'ils ne sont toujours que provisoires, les paradigmes, ainsi entendus, constituent une voie d'approche parfaitement légitime: dans la mesure où ils respectent l'oeuvre, ils la dévoilent. Il s'agit somme toute non de rejeter les paradigmes, mais de les nuancer.

Tout cela peut paraître évident, mais, paradoxalement, c'est précisément cette approche que Craven rejette. En effet, il se refère encore à Burke. Celui-ci avait clairement précisé sa position par rapport à la "tradition burckhardtienne" en affirmant: "it seems that all the apparently obvious characteristics of the Italian culture between 1420 and $1540 \ldots$ are not absolute characteristics, but that they can be saved, at the price of qualification" (p. 160).

Or Craven, de façon totalement inattendue, rejette cette façon de voir. La volonté de vouloir "sauver" les formules traditionnelles en les nuançant ou en leur donnant de nouvelles définitions, nous dit-il, est une attitude habituellement associée à la préservation des dogmes d'une foi religieuse, et la transposition de cette même attitude à l'égard d'une doctrine concernant une période de l'histoire montrerait à quel point cette doctrine est devenue un mythe ou une dogmatique.

Tel est le raisonnement de Craven (p. 160). On notera cependant que c'est lui qui, reprenant l'expression de R. Nisbet, parle de mythes et de dogmes à propos de la Renaissance. Il y a donc, de sa part également, excès de rhétorique. On notera d'ailleurs que si l'attitude consistant à nuancer les dogmes (ou à les "redéfinir"!) pour les mieux sauver, est en effet une attitude assez courante en dogmatique religieuse - ce n'est aussi qu'en ce domaine que l'on parle de "dogme" au sens premier et absolu du terme, et ce n'est donc également que par rapport à une telle dogmatique que cette attitude peut être jugée inacceptable ou contradictoire, car ce n'est qu'au sens absolu du terme que le dogme n'admet pas de nuance. On notera encore que le "mythe" de Pic ne naît pas seulement des exagérations évidentes dans l'interprétation de ses oeuvres, mais aussi de l'insistance même que l'on peut mettre à absolutiser ces exagérations. Ce que Craven semble méconnaître, au fond, c'est la possibilité qu'une vérité partielle soit présente, à la limite, dans chacune de ces nombreuses interprétations dont il se fait le critique. Il s'agirait alors avec prudence de reconstruire une image plausible à partir des éléments épars.

Craven s'interdit cette voie car le rejet des interprétations critiquées se fait aussi absolu que ces interprétations elles-mêmes. Dès lors, le déblayage effectué, il ne reste rien, ou presque rien, de Pic: réduit à toutes les orthodoxies de son temps, Pic devient anodin, parfaitement quelconque: "Pico is surprisingly unremarkable" (p. 129). ${ }^{7}$

Il est exact, comme Craven le signale à différentes reprises, que Pic ne 


\section{6 / Renaissance and Reformation}

prétendait proposer aucune nouvelle doctrine, qu'elle soit philosophique ou religieuse, il répète souvent sa conviction et son intention d'être parfaitement orthodoxe et s'il revendique une originalité ce n'est, chaque fois, que l'originalité d'une nouvelle approche, d'une nouvelle méthode, jamais celle d'un nouveau contenu.

Tout cela est exact et très bien observé par Craven, et pourtant, tout compte fait, son livre déçoit car, sa critique ayant visé juste, Craven en reste là, au milieu des disjecta membra de ce qui fut Pic. Reconnaissons toutefois qu'il nous avait prévenu: "it is not my intention to advance any new comprehensive view of his work as a whole" (p. 4). Pourtant, il semble qu'une telle vue d'ensemble était à portée de la main. C'est ce que j'espère pouvoir montrer en une autre occasion. Je signale seulement que le sous-titre même de l'ouvrage reprend et confirme une de ces ambiguités qui font partie du mythe mirandolien, un de ces "signaux routiers" fallacieux que Craven se propose d'arracher (p. 5): dans quelle mesure, ou dans quel sens, Pic était-il donc philosophe?

$$
* * *
$$

Ce compte rendu est sommaire. J'ai tenté de rendre l'essentiel de la pensée de l'auteur, en me méfiant déformations des inhérentes à ce genre d'entreprise, mais je n'ai pu rendre justice à toute la somme d'érudition et à l'excellente documentation qui entrent dans la composition d'un tel livre. J'ai tenu à en critiquer, avec vigueur, les conclusions finales en ce qu'elles ont d'extrème. J'espère néanmoins avoir fait ressortir l'importance de cet ouvrage, dont toute étude de Pic devra désormais tenir compte.

Ceci dit, le livre est présenté avec le soin habituel de la maison Droz. Quelques coquilles subsistent, mais elles ne nuisent en général pas à la lecture (signalons cependant un be gênant au lieu d'un the, p. 160, 1. 22).

Par souci de précision et voulant être systématique W. Craven procède avec ordre: les interprétations données à l'oeuvre de Pic sont regroupées par thèmes (l'homme, l'homme et Dieu, syncrétisme et universalisme, rationalisme et gnosticisme) ou centrées autour des "thèses condamnées" et des Disputationes. La méthode, quoique exhaustive, n'est pas sans inconvénient: les mêmes auteurs, proposant les mêmes interprétations fondamentales, à partir des mêmes points de vue, reviennent tour à tour, et comme Craven leur donne chaque fois largement la parole avant de les soumettre à sa critique - ce qui en soi est excellent - les répétitions, parfois textuelles, sont nombreuses. Elles étaient probablement inévitables, mais elles font d'autant plus regretter l'absence d'un bon index qui non seulement permettrait de retracer plus facilement tel ou tel thème, de retrouver telle référence à tel auteur - ce qui m'aurait été très utile! - mais en outre ferait probablement ressortir d'autres convergences que celles mises en lumière par l'auteur. L'absence de cet index est d'autant plus curieuse que l'auteur remercie nommément la collaboratrice qui l'avait préparé: il aura donc dû se perdre en cours de route, ce qui est fâcheux. 
Je note en contre-partie que la bibliographie est excellente puisque, divisée en trois sections, elle ne se limite pas à donner la liste des oeuvres de Pic, de leurs traductions et des études lui consacrées, mais qu'elle contient une solide section concernant les études rinascimentales en général ainsi que, pour terminer, quelques bons titres en historiographie.

Deux détails encore. L'auteur loue Giovanni Di Napoli pour l'attention avec laquelle celui-ci a lu les textes de Pic "even to the point of noting that there were only 899 theses" (p. 18). Or, il y a bien 900 thèses. Di Napoli a sans doute été induit en erreur par l'intitulé de la dernière section qui annonce LXXI thèses cabalistiques, alors qu'elle en contient 72.

L'autre point concerne la date de parution de l'Apologie. Innocent VIII était persuadé que Pic s'était rendu coupable de parjure car il aurait publié l'Apologie après avoir prêté serment de soumission, le 31 juillet 1487. Craven signale que la page-titre porte la date du 14 mai 1487, ce qui ne donnerait qu'un terminus a quo, (p. 73). En réalité cette date apparaît en dernière ligne de la dernière page, et, à moins d'un faux caractérisé, il faudrait donc y voir un terminus ad quem, ce qui exonorerait Pic de toute duplicité.

\section{Université de Sherbrooke}

\section{Notes}

1 Emst Bloch, La Philosophie de la Renaissance, traduit de l'allemand par P. Kamnitzer. Paris: Payot, 1974, p. 14-16.

2 Lidia Braghina, "Considerazioni sul pensiero morale di Pico," in L'Opera e il pensiero di Giovanni Pico della Mirandola nella storia dell' Umanesimo. Firenze: Istituto nazionale di Studi sul Rinascimento, 1965. (Vol. I: Relazioni) Vol. II: Comunicazioni, p. 17-34 (en particulier p. 31).

3 Eug. Garin, La cultura filosofica del Rinascimento italiano. Firenze: Sansoni, p. 178.

4 de Lubac, Pic de la Mirandole. Paris: Aubier-Montaigne, 1974.

5 Un cas particulièrement délicat -et que ne signale pas Craven- est celui du célèbre passage de l'Oratio où Pic exalte en l'homme cette capacité de dégénérescence et de régénérescence qui lui serait propre, et par laquelle il dépasserait en dignité les anges eux-mêmes. Le texte latin y oppose le passif regenerari à l'actif degenerare, et de Lubac (op. cit., p. 171) souligne que ce passif rend irrecevable toute interprétation pélagienne que l'on voudrait donner à la conception mirandolienne de la liberté. J'ajoute que, selon le Quicherat, regenerati est précisément le terme par lequel Isidore signifie les baptisés. Or, dans la traduction qu'il donne de ce passage, Cassirer, qui se refère à Burckhardt, oblitère ce contraste et ce sens en traduisant degenerare par entarten (dégrader) et regenerari par Wiedergebären (mettre au monde à nouveau). -E. Cassirer, Individuum und Kosmos in der Philosophie der Renaissance. LeipzigBerlin: B.G. Teubner, 1927, p. 90.

Garin va plus loin puisque sa traduction en langue italienne, pourtant en regard du texte latin, porte degenerare et regenerare, l'un et l'autre à l'actif: vision prométhéenne de l'homme capable de se régénérer soi-même. -G. Pico della Mirandola, De Hominis Dignitate, Heptaplus, De ente et uno, e scritti vari a cura di Eugenio Garin. Firenze: Valecchi ed. 1942. Reconnaissons cependant avec Craven que Garin "has moderated his views considerably over the intervening years" (p. 6).

6 Un trouvera un exemple parfait de ce "parochialism" dans la présentation d'une édition récente du Prince de Machiavel.

S'autorisant de cette seule citation signalée plus haut, en laquelle Burckhardt avait cru pouvoir résumer toute la pensée mirandolienne, l'auteur ne voit en Pic que le porte-parole d'une "seconde forme d'humanisme," dont "nous (Québécois) sommes les héritiers" (p. 48-49). Pic y est dépeint comme le 


\section{8 / Renaissance and Reformation}

grand libérateur luttant contre l'oppression cléricale, préfigurant ainsi le combat contre le clergé québécois de monseigneur Bourget, pour aboutir à Cité libre et aux Nègres blancs d'Amérique. Tout cela est d'ailleurs tellement conforme à la mythologie québécoise du moment, que le pauvre Pic se trouve en quelque sorte mythifié au carré ... .

(Cf. N. Machiavel. Le Prince et autres écrits politiques, traduction par J.V. Péries revue par Philippe Ranger, présentation, chronologie et notes par Philippe Ranger. Montréal: L'Hexagone/Minerve, 1982).

7 Craven fait cette remarque à propos des idées religieuses de Pic, mais chacune de ses analyses aboutit à un post-mortem similaire. 